\title{
Vagal-Immune Interactions in the Control of Hypertension
}

\author{
Renata M. Lataro', Helio C. Salgado2*
}

'Department of Physiological Sciences, Center of Biological Sciences, Federal University of Santa Catarina, Florianópolis, Santa Catarina, Brazil ${ }^{2}$ Department of Physiology, Ribeirão Preto Medical School, University of São Paulo, Ribeirão Preto, São Paulo, Brazil

\section{Article Info}

\section{Article Notes}

Received: September 20, 2018

Accepted: December 24, 2018

\section{${ }^{*}$ Correspondence:}

Dr. Helio C. Salgado, MD, Ph.D, Department of Physiology, Ribeirão Preto Medical School, University of São Paulo, Av. Bandeirantes 3900, 14049-900 Ribeirão Preto, SP, Brazil; Telephone No: 55-16-33153201; E-mail: hcsalgado@fmrp.usp.br.

(C) 2018 Salgado HC. This article is distributed under the terms of the Creative Commons Attribution 4.0 International License

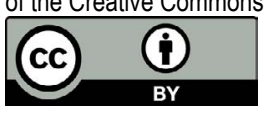

\section{ABSTRACT}

There is a consensus in the literature that the autonomic dysfunction in arterial hypertension involves the increase of sympathetic activity and, also, reduction of vagal tone. In the last years, the role played by inflammation in the development of hypertension and target organs injuries, such as heart and blood vessels, has been emphasized. Although the clinical importance of sympathetic hyperactivity and its treatment of arterial hypertension is recognized, the therapeutic benefit of increasing parasympathetic activity in hypertensive patients still requires an in-depth investigation. The increased vagal activity may produce beneficial effects on cardiovascular modulation and inflammation, preventing target organ damage. Parasympathetic neurotransmission can be improved by the inhibition of acetylcholinesterase action. Anticholinesterase agents prevent the hydrolysis of acetylcholine by acetylcholinesterase, prolonging its availability within the cholinergic cleft. This article will highlight the key concepts of the cardiac autonomic imbalance and the increase of acetylcholine availability under inflammation and control of arterial hypertension. In conclusion, significant evidence exists associating the reduction of parasympathetic activity and the occurrence of inflammation involving the pathophysiology of hypertension; suggesting that the improvement of vagal activity by the increased availability of acetylcholine has a remarkable potential for the therapeutic intervention on arterial hypertension.

\section{Introduction}

A recent American Heart Association report shows that approximately 86 million of US adults exhibit arterial hypertension ${ }^{1}$. Globally, the rate of elevated systolic blood pressure (BP) increased substantially between 1990 and $2015^{2}$. A rise in arterial pressure associated with weight gain, lifestyle factors, reduced physical activity, or advancing age with a strong family history of hypertension would suggest the diagnosis of primary (essential) hypertension ${ }^{3}$. On the other hand, secondary hypertension is elevated BP that results from an underlying, identifiable, often correctable cause ${ }^{4}$. Arterial hypertension produces functional and structural abnormalities in target organs, such as the heart, kidneys, brain and blood vessels, and correlates positively with the risk of occurrence of adverse cardiovascular events leading to death ${ }^{1}$. Hypertension maintains an independent association with events such as sudden death, stroke, acute myocardial infarction, heart failure, peripheral arterial disease, and chronic, fatal and nonfatal renal disease ${ }^{3}$. Although hypertension can be treated, 8 to $17 \%$ of the 900 million people with hypertension worldwide are resistant to treatment ${ }^{2,3}$. Therefore, there is still a need for the determination of an effective treatment to control the BP of these patients.

\section{Autonomic Dysfunction, Inflammation and Hypertension}

Arterial hypertension includes, in its multifactorial etiology, an abnormality of the autonomic modulation of $\mathrm{BP}$, characterized by 
sympathetic activation, which is accompanied by an inhibition of parasympathetic activity ${ }^{6-8}$. Data collected from experimental animals and humans support the hypothesis that sympathetic neural mechanisms are involved in the development, maintenance and progression of arterial hypertension ${ }^{9}$. The evaluation of sympathetic cardiovascular activity in humans has shown that the increase of sympathetic activity occurs in hypertensive patients, and that the magnitude of this elevation is proportional to the severity of the arterial hypertension ${ }^{6}$. In addition, when the hypertensive state is associated with the presence of target organ damage, the magnitude of sympathetic activation appears to be potentiated ${ }^{10,11}$. Patients with arterial hypertension have plasma levels of noradrenaline $25-30 \%$ higher than normal individuals with the same age ${ }^{6}$. These patients also have elevated heart rate (HR) and increased peripheral resistance ${ }^{6,12}$, which also reflect increased sympathetic activity ${ }^{13}$. Clinical evidence has also shown that sympathetic hyperactivity is related to target organ damage in arterial hypertension, including cardiac hypertrophy, left ventricular diastolic dysfunction, heart failure, as well as structural and functional abnormalities, frequently detected in large, medium and small arteries ${ }^{9}$. However, elevated HR values are not only determined by the sympathetic hyperactivity, but is also influenced by the reduced parasympathetic activity ${ }^{13}$. Reduction of the vagal tone is associated with increased risk of cardiovascular disease ${ }^{14}$. The high frequency of the HR oscillatory component is reduced in hypertensive patients, indicating a deficient cardiac parasympathetic modulation ${ }^{14}$. Reduction of baroreflex responses mediated by the vagus nerve ${ }^{15,16}$, and attenuation of HR recovery after physical exercise in hypertensive patients are also observed, indicating a reduction of the parasympathetic control of the $\mathrm{HR}^{7}$. Hence, there is a consensus in the literature that the autonomic dysfunction in arterial hypertension, in humans and experimental animals, involves the increase of sympathetic activity, but also the reduction of the vagal tone $\mathrm{e}^{6-8}$. Although the clinical importance of sympathetic hyperactivity and its management in arterial hypertension is well recognized, the therapeutic benefit of increasing parasympathetic activity in hypertensive patients remains to be in-depth investigated. The increase in parasympathetic activity may produce beneficial effects on autonomic cardiovascular control, inflammation, and prevention of target organ damage $\mathrm{e}^{17}$.

In the last years, the role played by inflammation in the development of arterial hypertension and target organs injury has gained remarkable importance ${ }^{18-20}$. Clinical evidence has shown that arterial hypertension is associated with increased plasma concentrations of inflammatory mediators combined with vascular inflammation ${ }^{18,20}$. Hypertensive patients show increased levels of circulating monocytes, lymphocytes and proinflammatory cytokines, such as tumor necrosis factor $\alpha$ (TNF- $\alpha)$, interleukin (IL) 6 and C-reactive protein $^{21-23}$. Vascular inflammation is characterized by an accumulation of immune cells such as macrophages, monocytes, dendritic cells, B and T lymphocytes; moreover, increased expression of proinflammatory cytokines and cell adhesion molecules in different layers of the vascular wall of patients or hypertensive rodents have been described as well ${ }^{19}$. These factors induce extracellular matrix deposition, smooth muscle hypertrophy, and endothelial dysfunction, contributing to the development and maintenance of arterial hypertension ${ }^{24}$. Furthermore, activation of the immune system also increases the sympathetic activity and promotes renal damage ${ }^{25}$. In rats, the intracerebroventricular infusion of IL-1 $\beta$, a proinflammatory cytokine, activated the sympathetic nervous system and increased $\mathrm{BP}^{26,27}$. Besides, interferon- $\gamma$ and IL-1 seems to be critical for the development of renal injury in experimental hypertension induced by angiotensin II in mice ${ }^{25,28}$. It has also been reported the involvement of TNF- $\alpha$ in sodium and water retention in the development of hypertension induced by angiotensin II infusion ${ }^{29,30}$. In Dahl salt-sensitive rats, IL- 6 has been associated with renal inflammation and injury ${ }^{31}$. Of note, a balance between pro and anti-inflammatory factors may determine the degree of inflammation. In this regard, added to the activation of proinflammatory factors, the deficit in anti-inflammatory mechanisms has been described in experimental models of hypertension ${ }^{25,32,33}$. Regulatory T lymphocytes suppress the immune response, inhibiting the proinflammatory effects of other lymphocytes, macrophages, dendritic cells and neutrophils ${ }^{25}$. The action of the regulatory $\mathrm{T}$ lymphocytes can be mediated by the production of anti-inflammatory cytokines, such as IL-10 and transforming growth factor $\beta$ (TGF- $\beta)^{25,32}$. Dahl salt-sensitive hypertensive rats exhibit dysfunctional regulatory $\mathrm{T}$ lymphocytes in their vascular beds, producing low levels of immune suppressive mediators TGF- $\beta$ and IL-10, increasing the inflammatory response $^{32}$. Administration of adoptive regulatory $\mathrm{T}$ lymphocytes prevented BP elevation, vascular oxidative stress, vascular inflammation, endothelial dysfunction, and cardiac hypertrophy produced by the administration of angiotensin II in mice ${ }^{33,34}$. Collectively, these observations indicate that inflammation contributes to the pathophysiology of hypertension, eliciting the impairment of natriuresis, dysfunctional vascular relaxation, and overactivity of the sympathetic nervous system. Hence, the immune system can be a new target for the treatment of arterial hypertension.

\section{Parasympathetic Activity Improvement in Arterial Hypertension}

In the last decade, the role played by the vagus nerve as a modulator of the immune response has gained paramount attention ${ }^{35,36}$. The inflammatory reflex is a neural circuit, 
with afferent and efferent pathways, organized in a reflexive manner to regulate immune responses and inflammation ${ }^{35,37}$. The activity of the afferent (sensory) arm is modulated by cytokines or other inflammatory ends products present in the tissues ${ }^{37}$. The afferent information is carried to the brainstem nuclei that control the efferent neural signals ${ }^{38}$. The efferent arm of the inflammatory reflex is called "the cholinergic anti-inflammatory pathway" 37 . The efferent vagus nerve fibers provide a conduit of brainto-immune communication for controlling TNF and other pro-inflammatory cytokine production through the release of acetylcholine $\mathrm{s}^{35,38}$. The cytokine expression is inhibited by molecular mechanisms that require the transduction of the signal via alpha 7 nicotinic acetylcholine receptor $(\alpha 7 \mathrm{nAChR})$ expressed on macrophages, dendritic cells and other immune cells ${ }^{35,36,38}$. Therefore, acetylcholine attenuates the tissue inflammation by reducing the release of proinflammatory cytokines. Electrical vagus nerve stimulation suppressed hepatic, cardiac and circulating TNF levels, while vagotomy abolished these effects during endotoxemia in mice ${ }^{38,39}$. In addition, animals with deficient vagal activity or $\alpha-7 \mathrm{nAChR}$ expression are more susceptible to inflammatory changes due to high production of cytokines $^{38}$. Clinical studies demonstrated that when the parasympathetic activity is attenuated, the inflammation is more pronounced ${ }^{40}$. Vagus nerve stimulation inhibited the release of cytokines and promoted considerable protection to tissue injury in experimental models of arthritis, sepsis, and ischemia-reperfusion ${ }^{36,39}$. However, there are few data showing beneficial effects of increased parasympathetic activity in arterial hypertension. Reduction of vagal tone, assessed by the HR response to atropine administration, is associated with the deficiency in $\alpha-7 \mathrm{nAChR}$ expression and acetylcholine vesicle transporter in the heart, kidneys, and aorta of spontaneously hypertensive rats $(\mathrm{SHR})^{17}$. Furthermore, the treatment of SHR with an $\alpha-7 \mathrm{nAChR}$ agonist reduced the tissue levels of proinflammatory cytokines and prevented the target organ damage, in the heart, kidneys, and aorta ${ }^{17}$. The stimulation of neurons residing in the dorsal vagal motor nucleus reduced the SHR's arterial pressure ${ }^{41}$. Other approaches used to increase vagal modulation in hypertension, such as baroreflex activation therapy, renal sympathetic denervation, and direct vagal nerve stimulation, also produced beneficial effects for this pathological condition ${ }^{42}$. Despite the positive results, all of these methods are invasive, while the pharmacological approaches have the advantage of being noninvasive.

The increase of parasympathetic activity can be obtained by acetylcholinesterase inhibition. The parasympathetic efferent control is mediated by acetylcholine, whereas the acetylcholinesterase blockade increases the availability of acetylcholine in the synaptic cleft, increasing vagal activity ${ }^{43}$. Anticholinesterase agents such as donepezil and pyridostigmine have been used in experimental designs to increase parasympathetic function ${ }^{44}$. Previous studies conducted in our laboratory demonstrated that chronic treatment with pyridostigmine preserved the cardiac autonomic balance in heart failure rats, increasing the parasympathetic and reducing the cardiac sympathetic tone ${ }^{45}$. Chronic treatment with pyridostigmine also prevented cardiac remodeling, improved cardiac function and attenuated the heart failure progression in rats ${ }^{45}$. Pyridostigmine is hydrosoluble, and due to its quaternary ammonium structure does not cross the blood-brain barrier, preventing its action upon the central nervous system ${ }^{43}$. Therefore, the pyridostigmine effect is expressed only peripherally ${ }^{43}$. On the other hand, donepezil is an anticholinesterase agent that crosses the blood-brain barrier and has a high degree of selectivity for acetylcholinesterase ${ }^{46}$. Donepezil has a long half-life and is used in the Alzheimer's disease treatment ${ }^{46,47}$. In heart failure rats, donepezil increased cardiac parasympathetic modulation, preventing sympathetic activation and cardiac remodeling ${ }^{44}$. Patients with Alzheimer's disease that were treated for 1-month with donepezil showed reduced levels of oncostatin M, 6 and 1 in the peripheral blood mononuclear cells, suggesting a possible anti-inflammatory effect of this centrally acting anti-acetylcholinesterase agent ${ }^{47}$. Antiinflammatory effects of donepezil were also observed in apolipoprotein E-knockout mice that showed suppressed expression of monocyte chemoattractant protein-1 and TNF- $\alpha$ in the aorta ${ }^{48}$. Our group demonstrated that longterm (16 weeks) administration of donepezil, in SHR, attenuated the development of hypertension, improved the cardiovascular autonomic modulation, reduced the plasma proinflammatory cytokine levels (TNF- $\alpha$, IL-6, and IFN- $\gamma$ ) and prevented the cardiac remodeling ${ }^{49}$. However, pyridostigmine, an anti-acetylcholinesterase agent that does not cross the blood-brain barrier, despite its positive effect on cardiac vagal tone, was unable to reduce the high blood pressure and plasma cytokine levels in hypertensive rats $^{49}$. These results indicate that the antihypertensive effect produced by donepezil probably involves an antiinflammatory effect. Cytokines produced peripherally and also in the brain contribute to the pathophysiology in arterial hypertension ${ }^{18}$. Shi et $\mathrm{al}^{50}$ demonstrated that intracerebroventricular infusion of minocycline, a broadspectrum antibiotic from the tetracycline family, decreased the expression of proinflammatory cytokines in the paraventricular nucleus, and reduced the BP in hypertensive rats. Hence, these data suggest that the increased availability of acetylcholine, induced by donepezil administration, may produce anti-inflammatory effects in essential areas of the central nervous system for controlling the BP, producing its antihypertensive effects. Taken together, these data provide evidence that the blockade of acetylcholinesterase within the central nervous system may be a potential target for the treatment of arterial hypertension. 


\section{Conclusions and Future Directions}

In conclusion, there is significant evidence associating the reduction of parasympathetic activity as well as inflammation in the assessment of the pathophysiology of arterial hypertension; this evidence suggests that the improvement of vagal function by the increased availability of acetylcholine has a substantial potential for the therapeutic interventions in arterial hypertension.

\section{References}

1. Benjamin EJ, Blaha MJ, Chiuve SE, et al. American Heart Association Statistics Committee and Stroke Statistics Subcommittee. Heart disease and stroke statistics-2017 update: a report from the American Heart Association. Circulation. 2017; 135: e146-e603.

2. Forouzanfar MH, Liu P, Roth GA, et al. Global burden of hypertension and systolic blood pressure of at least 110 to $115 \mathrm{~mm} \mathrm{Hg}, 1990-2015$ JAMA. 2017; 317: 165-182.

3. Whelton PK, Carey RM, Aronow WS, et al. ACC/ AHA/ AAPA/ ABC/ ACPM/ AGS/ APhA/ ASH/ ASPC/ NMA/ PCNA Guideline for the prevention, detection, evaluation, and management of high blood pressure in adults: A Report of the American College of Cardiology/ American Heart Association Task Force on Clinical Practice Guidelines. J Am Coll Cardiol. 2018; 71(19): e127-e248.

4. Onusko E. Diagnosing secondary hypertension. Am Fam Physician 2003; 67(1): 67-74.

5. Carey RM. Resistant hypertension. Hypertension. 2013; 61: 746-750.

6. Grassi G, Seravalle G, Quarti-Trevano F. The 'neuroadrenergic hypothesis' in hypertension: current evidence. Exp Physiol. 2010; 95 581-658.

7. Polónia J, Amaral C, Bertoquini S, et al. Attenuation of heart rate recovery after exercise in hypertensive patients with blunting of the night time blood pressure fall. Int J Cardiol. 2006; 106(2): 238-243.

8. Edwards KM, Wilson KL, Sadja J, et al. Effects on blood pressure and autonomic nervous system function of a 12-week exercise or exercise plus DASH-diet intervention in individuals with elevated blood pressure. Acta Physiol. 2011; 203(3): 343-50.

9. Grassi G, Seravalle G, Dell'oro R, et al. Sympathetic mechanisms, organ damage, and antihypertensive treatment. Curr Hypertens Rep. 2011; 13(4): 303-308.

10. Schlaich MP, Kaye DM, Lambert E, et al. Relation between cardiac sympathetic activity and hypertensive left ventricular hypertrophy. Circulation. 2003; 108: 560-565.

11. Burns J, Sivananthan MU, Ball SG, et al. Relationship between central sympathetic drive and magnetic resonance imaging-determined left ventricular mass in essential hypertension. Circulation. 2007; 115: 1999-2005.

12. Fisher JP, Fadel PJ. Therapeutic strategies for targeting excessive central sympathetic activation in human hypertension. Exp Physiol. 2010; 95: 572-580.

13. Julius S. Tachycardia in hypertension: a saga of progress despite prejudice, confusion, and inertia. Prog Cardiovasc Dis. 2009; 52: 2630

14. Thayer JF, Lane RD. The role of vagal function in the risk for cardiovascular disease and mortality. Biol Psychol. 2007; 74: 224-242.

15. Petretta M, Marciano F, Bianchi V, et al. Power spectral analysis of heart period variability in hypertensive patients with left ventricular hypertrophy. Am J Hypertens. 1995; 8: 1206-1213.

16. Malliani A, Pagani M, Lombardi F, et al. Spectral analysis to assess increased sympathetic tone in arterial hypertension. Hypertension. 1991; 17 (suppl III): III36-III42.

17. Li DJ, Evans RG, Yang ZW, et al. Dysfunction of the cholinergic antiinflamatory pathway mediates organ damage in hypertension. Hypertension. 2011; 57(2): 298-307.

18. Zubcevic J, Waki H, Raizada MK, et al. Autonomic-immune-vascular interaction: an emerging concept for neurogenic hypertension. Hypertension. 2011; 57(6): 1026-1033.

19. McMaster WG, Kirabo A, Madhur MS, et al. Inflammation, immunity, and hypertensive end-organ damage. Circ Res. 2015; 116(6): 1022-1033.

20. Stocker SD, Kinsman BJ, Sved AF. Recent Advances in neurogenic hypertension: dietary salt, obesity, and inflammation. Hypertension. 2017 [Epub ahead of print].

21. Cachofeiro V, Miana $\mathrm{M}$, de las Heras $\mathrm{N}$, et al. Inflammation: a link between hypertension and atherosclerosis. Curr Hypertens Rev. 2009; 5: 40-48

22. Fliser D, Buchholz $\mathrm{K}$, Haller $\mathrm{H}$, et al. Antiinflammatory effects of angiotensin II subtype 1 receptor blockade in hypertensive patients with microinflammation. Circulation. 2004; 110: 1103-1107.

23. Blake GJ, Ridker PM. Novel clinical markers of vascular wall inflammation. Circ Res. 2001; 89: 763-741.

24. Guzik TJ, Touyz RM. Oxidative stress, inflammation, and vascular aging in hypertension. Hypertension. 2017; 70(4): 660-667.

25. Rodriguez-Iturbe B, Pons H, Johnson RJ. Role of the immune system in hypertension. Physiol Rev. 2017; 97(3): 1127-1164.

26. Kannan H, Tanaka Y, Kunitake T, et al. Activation of sympathetic outflow by recombinant human interleukin-1 in conscious rats. Am J Physiol. 1996; 270: R479-R485.

27. Lu Y, Chen J, Yin X, et al. Angiotensin II receptor 1 involved in the central pressor response induced by interleukin-1 in the paraventricular nucleus. Neurol Res. 2009; 31: 420-424.

28. Crowley SD, Song YS, Lin EE, et al. Lymphocyte responses exacerbate angiotensin II-dependent hypertension. Am J Physiol Regul Integr Comp Physiol. 2010; 298: R1089-R1097.

29. Sriramula S, Haque M, Majid DS, et al. Involvement of tumor necrosis factor- $\alpha$ in angiotensin II-mediated effects on salt appetite, hypertension, and cardiac hypertrophy. Hypertension. 2008; 51: 1345-1351.

30. Zhang J, Patel MB, Griffiths R, et al. Tumor necrosis factor- $\alpha$ produced in the kidney contributes to angiotensin II-dependent hypertension. Hypertension. 2014; 64: 1275-1281.

31. Hashmat S, Rudemiller N, Lund $\mathrm{H}$, et al. Interleukin-6 inhibition attenuates hypertension and associated renal damage in Dahl saltsensitive rats. Am J Physiol Renal Physiol. 2016; 311: F555-F561.

32. Schiffrin EL. T lymphocytes: a role in hypertension. Curr Opin Nephrol Hypertens. 2010; 19(2): 181-186.

33. Barhoumi T, Kasal DA, Li MW, et al. T Regulatory lymphocytes prevent angiotensin II-induced hypertension and vascular injury. Hypetension. 2011; 57(3): 469-476.

34. Kvakan H, Kleinewietfeld M, Qadri F, et al. Regulatory T cells ameliorate angiotensin II-induced cardiac damage. Circulation. 2009; 119: 2904-2912.

35. Chavan SS, Pavlov VA, Tracey KJ. Mechanisms and therapeutic relevance of neuro-immune communication. Immunity. 2017; 46(6): 927-942.

36. PavlovVA, Tracey KJ. Neural regulation of immunity: molecularmechanisms and clinical translation. Nat Neurosci. 2017; 20(2): 156-166. 26.

37. Tracey KJ. The inflammatory reflex. Nature. 2002; 420: 853-859. 
38. Tracey KJ. Understanding immunity requires more than immunology. Nature Immunol. 2010; 11: 561-564.

39. Bernik TR, Friedman SG, Ochani M, et al. Cholinergic antiinflammatory pathway inhibition of tumor necrosis factor during ischemia reperfusion. J Vasc Surg. 2002; 36(6): 1231-1236.

40. Huston JM, Tracey KJ. The pulse of inflammation: heart rate variability, the cholinergic anti-inflammatory pathway and implications for therapy. J Intern Med. 2011; 269(1): 45-53.

41. Moreira TS, Antunes VR, Falquetto B, et al. Long-term stimulation of cardiac vagal preganglionic neurons reduces blood pressure in the spontaneously hypertensive rat. J Hypertens. 2018. [Epub ahead of print]

42. Petkovich BW, Vega J, Thomas S. Vagal modulation of hypertension. Curr Hypertens Rep. 2015; 17(4): 532.

43. Taylor P. In: Goodman and Gilman's Pharmacological Basis of Therapeutics. Ed. Hardman JG, Limbird LE: 175-192, McGraw-Hill, New York, 2001.

44. Okazaki Y, Zheng C, Li M, et al. Effect of the cholinesterase inhibitor donepezil on cardiac remodeling and autonomic balance in rats with heart failure. J Physiol Sci. 2010; 60(1): 67-74.
45. Lataro RM, Silva CA, Fazan R Jr, et al. Increase in parasympathetic tone by pyridostigmine prevents ventricular dysfunction during the onset of heart failure. Am J Physiol Regul Integr Comp Physiol. 2013; 15; 305(8): R908-R916.

46. Sugimoto H. Donepezil hydrochloride: a treatment drug for alzheimer's disease. Chem Rec. 2001; 1(1): 63-73.

47. Reale $\mathrm{M}$, Iarlori $\mathrm{C}$, Gambi $\mathrm{F}$, et al. Acetylcholinesterase inhibitors effects on oncostatin- $M$, interleukin- 1 beta and interleukin- 6 release from lymphocytes of Alzheimer's disease patients. Exp Gerontol. 2005 ; 40: 165-171.

48. Inanaga $\mathrm{K}$, Ichiki $\mathrm{T}$, Miyazaki $\mathrm{R}$, et al. Acetylcholinesterase inhibitors attenuate atherogenesis in apolipoprotein E-knockout mice. Atherosclerosis. 2010; 213: 52-58.

49. Lataro RM, Silva CA, Tefé-Silva C, et al. Acetylcholinesterase Inhibition Attenuates the Development of Hypertension and Inflammation in Spontaneously Hypertensive Rats. Am J Hypertens. 2015; 28(10): 1201-1208.

50. Shi P, Diez-Freire C, Jun JY, et al. Brain microglial cytokines in neurogenic hypertension. Hypertension. 2010; 56: 297-303. 